\title{
Editorial
}

Digestion

\section{Autoimmune Pancreatitis in Germany: Rare but Relevant}

\author{
Golo Petzold Volker Ellenrieder Albrecht Neesse \\ Department of Gastroenterology and Gastrointestinal Oncology, University Medical Centre Göttingen, \\ Göttingen, Germany
}

Autoimmune pancreatitis (AIP) is an increasingly recognized fibro-inflammatory disease of the pancreas, characterized by distinct histopathological and clinical features. AIP-type 1 not only affects the pancreas but frequently involves a multiorgan syndrome (kidney, salivary glands, biliary tree, retroperitoneum, lymph nodes) that is referred to as IgG-4-related disease. It occurs mostly in elderly men with painless jaundice and is frequently accompanied by elevated IgG-4 serum levels. The histopathological form is described as lymphoplasmic sclerosing pancreatitis, but diagnosis can often be made without histological sampling if IgG-4 serum levels are sufficiently high [1].

In contrast, AIP-type 2 is a pancreas-specific inflammation that often only focally involves the pancreas and can easily be misdiagnosed as pancreatic cancer. It occurs more often in younger patients with painful pancreatitis episodes and associated inflammatory bowel disease. Other organ involvement does not occur and IgG-4 levels are typically not elevated. On histology, AIP-type 2 is termed idiopathic duct centric pancreatitis, and sufficient biopsy material is often difficult to obtain using minimally invasive techniques [2].

Usually, AIP responds well to oral steroids initially; however, in some patients there is a relapse, especially in those with AIP-type 1, and hence low-dose steroid main-

\section{KARGER}

๑) 2017 S. Karger AG, Basel

E-Mail karger@karger.com

www.karger.com/dig tenance or alternative immunosuppressive strategies are required [3].

To standardize and facilitate the challenging diagnosis of AIP, especially with regard to the life-threatening differential diagnosis of pancreatic cancer, international consensus diagnostic criteria have been developed that entail 5 diagnostic key features: pancreatic imaging, steroid response, serology, other organ involvement, and histology that are categorized in levels 1 and 2 evidences.

Especially in western countries, the incidence and prevalence of AIP is largely unknown.

In Japan, a recent survey suggests a prevalence rate of about 4 per 100,000 individuals, and an annular incidence rate of 1.4 per 100,000 [4].

In a clinical routine, acute or chronic pancreatitis will mostly be caused by chronic alcohol abuse or biliary pathologies. However, it is extremely important to know when to look for rare causes of pancreatitis, such as AIP.

In this issue of Digestion, Schneider et al. [5] present a retrospective cross-sectional study in $(n=704)$ patients ( $n=373$ with alcoholic pancreatitis, and $n=331$ with non-alcoholic pancreatitis) from a tertiary referral hospital in the southwest of Germany. The authors analyze 2 different cohorts, the retrospective pancreas cohort (RPC; $n=534$ patients) and the pancreas clinic cohort (PCC; $n=170$ ) for features of AIP. In the RPC, preva- 
lence of AIP was $5.9 \%$ in patients with non-alcoholic pancreatitis, whereas no AIP was diagnosed in $(n=313)$ alcoholic pancreatitis cases. The PCC, which was more rigorously and systematically assessed for AIP features, showed a prevalence of $9.1 \%$ among patients with nonalcoholic pancreatitis, whereas only 1 case of AIP was diagnosed in alcoholic pancreatitis patients. For chronic pancreatitis, the prevalence of AIP was $6.5 \%$, which is in agreement with data obtained from Korea (5.4\%), Japan (4-8\%), and Italy (4-6\%). The estimated incidence for AIP was 0.29 per 100,000 population each year and lower than calculated for Asian countries. Notably, surgical interventions were undertaken in almost a third of patients $(7 / 24)$ due to initially suspected pancreatic cancer. Despite the obvious limitations of the retrospective study design, including the different patient cohorts with heterogenous data collection, and the fact that the study was performed by pancreatologists in a specialized referral center, that may encounter more cases of unexplained pancreatitis patients, the study provides valuable information for practicing physicians. It shows for the first time that AIP hardly occurs in patients with acute or chronic alcoholic pancreatitis. Furthermore, there is an ongoing debate whether AIP-type 1 is more frequent in Asia, whereas AIP-type 2 prevails in western countries. Comparing the 2 different cohorts (RPC: 1998-2008) and PCC (2009-2014), the frequency of AIP-type 2 increased from $8 \%$ (RPC) to $55 \%$ (PCC), suggesting that increasing experience and rigorous use of international consensus diagnostic criteria in the PCC may have increased the diagnosis of AIP-type 2. Therefore, it is important to remember that normal IgG-4 serum levels do not exclude the diagnosis of AIP, and AIP-type 2 requires histological evidence, unless typical imaging features, association with chronic inflammatory bowel disease or other autoimmune diseases, and response to steroids can be observed simultaneously. Whether the higher incidence of AIP-type 1 in Korea and Japan is a matter of different diagnostic approaches or genetic background is an ongoing debate. However, a prospective multicenter study from Japan showed that endoscopic ultrasonography (EUS)-guided fine needle aspiration was only able to diagnose AIP in 3 out of 38 patients (sensitivity of 7\%) [6]. This study highlights the current dilemma in the diagnosis of AIP-type 2 and, also explains why almost one third of patients with AIP underwent surgical procedures unnecessarily. Thus, future efforts should be aimed at improving the efficacy of EUSfine needle aspiration and EUS-guided trucut biopsy by developing better needle prototypes and choosing the best biopsy technique.

\section{Funding Sources}

The authors have no funding to declare.

\section{References}

1 Culver EL, Sadler R, Simpson D, Cargill T, Makuch M, Bateman AC, et al: Elevated serum IgG4 levels in diagnosis, treatment response, organ involvement, and relapse in a prospective IgG4-related disease UK cohort. Am J Gastroenterol 2016;111:733-743.

2 Hart PA, Levy MJ, Smyrk TC, Takahashi N, Abu Dayyeh BK, Clain JE, et al: Clinical profiles and outcomes in idiopathic duct-centric chronic pancreatitis (type 2 autoimmune pancreatitis): the mayo clinic experience. Gut 2016;65:1702-1709.
3 Mizuno N, Liyama T, et al; Research Committee of Intractable Pancreas Diseases in Japan: Randomised controlled trial of long-term maintenance corticosteroid therapy in patients with autoimmune pancreatitis. Gut 2017;66:487-494.

4 Kanno A, Masamune A, Okazaki K, Kamisawa T, Kawa S, Nishimori I, et al; Research Committee of Intractable Diseases of the Pancreas: Nationwide epidemiological survey of autoimmune pancreatitis in Japan in 2011. Pancreas 2015;44:535-539.
5 Schneider A, Michaely H, Weiss C, Hirth M, et al: Prevalence and incidence of autoimmune pancreatitis in the population living in the southwest of Germany. Digestion 2017; 96:187-198.

6 Morishima T, Kawashima H, Ohno E, Yamamura T, Funasaka K, Nakamura M, et al: Prospective multicenter study on the usefulness of EUS-guided FNA biopsy for the diagnosis of autoimmune pancreatitis. Gastrointest Endosc 2016;84:241-248. 